\title{
The Impact of Immunonutritional and Physical Status on Surgical Outcome After Pancreaticoduodenectomy in Elderly Patients
}

\author{
KEISHI SUGIMACHI ${ }^{1}$, TOMOHIRO IGUCHI ${ }^{1}$, YOHEI MANO ${ }^{1}$, TOMOHIRO NISHIJIMA ${ }^{2}$, \\ TOMONORI NAKANOKO $^{3}$, HIDEO UEHARA ${ }^{3}$, MASAHIKO SUGIYAMA ${ }^{3}$, \\ MITSUHIKO OHTA ${ }^{3}$, MASAHIKO IKEBE ${ }^{3}$, MASARU MORITA ${ }^{3}$ and YASUSHI TOH ${ }^{3}$ \\ Department of ${ }^{1}$ Hepatobiliary-Pancreatic Surgery, ${ }^{2}$ Geriatric Oncology Service, \\ ${ }^{3}$ Gastroenterological Surgery, National Hospital Organization Kyushu Cancer Center, Fukuoka, Japan
}

\begin{abstract}
Background/Aim: The aim of this study was to determine the significance of immunonutritional and physical index in the assessment of risk associated with pancreaticoduodenectomy (PD) in the elderly. Patients and Methods: This study enrolled 92 patients who underwent PD. They were divided into 2 groups: Group A included patients 79 years and younger $(n=79)$ and Group B patients 80 years and older $(n=13)$. Among 37 patients, physical function and body composition were also evaluated. Results: Significantly higher neutrophil-lymphocyte ratio, lower prognostic nutritional index (PNI), and controlling nutritional score were observed in Group B. Muscle strength and walking ability were significantly impaired in Group B, although there was no significant difference in body composition. Age was not correlated with the incidence of postoperative complications, overall survival or recurrencefree survival by univariate and multivariate analysis. Conclusion: PD is justified for the elderly, with acceptable morbidity and prognosis. However, immunonutritional status and physical function are significantly impaired; thus, appropriate case selection and active nutritional support are required for the elderly.
\end{abstract}

Pancreaticoduodenectomy (PD) is the only curative option for pancreatic head, distal biliary tract, and duodenal malignant tumors. The number of elderly patients who

Correspondence to: Keishi Sugimachi, MD, Ph.D., Department of Hepatobiliary-Pancreatic Surgery, National Hospital Organization Kyushu Cancer Center, 3-1-1 Notame, Minami-ku, Fukuoka 8111395, Japan. Tel: +81 925413231, Fax: +81 975428503, e-mail: ksugi@surg2.med.kyushu-u.ac.jp

Key Words: Pancreaticoduodenectomy, elderly, complication, nutrition, physical status. undergo PD is rising in Japan, where the elderly population is rapidly increasing as in many other countries. These patients are generally and potentially characterized by declining physiological functions, poor nutritional status, impaired physical activity and cognitive function. Elderly patients may have poorer prognoses compared to younger patients because of their increased risk of postoperative complications and fewer expected life years.

Perioperative management of patients undergoing PD has dramatically improved over the past few decades, but the morbidity rate still ranges from $20 \%$ to $50 \%(1,2)$. In the past, dismal early and long-term outcomes restricted PD to younger patients who did not have comorbid diseases (3). A recent cohort study reported that PD in elderly patients was still associated with significantly more postoperative complications and higher costs (4). However, on the contrary, a systematic literature review of 21 studies reported that PD was feasible in elderly patients, with acceptable morbidity and mortality rates (5). However, because of a high incidence of comorbid diseases, elderly patients are still generally considered high-risk patients for major surgery. Given these conflicting data, objective indicators for choosing the surgical indication for the elderly are strongly needed.

Preoperative malnutrition and sarcopenia are associated with a higher incidence of complications after PD (6-8), and preoperative administration of an immune-enhancing diet is reported to improve clinical outcomes in patients undergoing PD (9). Aging as well as underlying malignancy are associated with malnutrition and sarcopenia; therefore, assessment of the nutritional condition is critical to decide whether elderly patients should undergo surgery. Perioperative nutritional support and preoperative rehabilitation are beneficial for elderly patients who will undergo major abdominal surgery, and these practices can reduce the rates of surgical complications and mortality $(10,11)$. A number of indicators, including the neutrophil-to-lymphocyte ratio (NLR), the 
prognostic nutritional index (PNI), the Glasgow Prognostic Score (GPS), and the Controlling Nutritional Status (CONUT) score have been reported to be useful predictors of morbidity and mortality for various oncological surgeries (12-16). These parameters are universally correlated with clinical outcome with high accuracy among various diseases, and have the advantage of being calculated easily without any invasive methods. Therefore, we considered whether these indicators could be applied to evaluate which elderly patients are highrisk PD patients.

Given the importance of assessing patient-specific risk factors for PD-related complications or mortality in the elderly, we conducted a retrospective study to investigate the impact of immunonutritional and physical parameters among patients undergoing PD. Specifically, we hypothesized that these parameters are useful to establish effective and safe strategies to prepare elderly patients for major abdominal surgery.

\section{Patients and Methods}

Patients. A retrospective cohort study was performed with 92 consecutive patients who underwent PD at a single institute (Kyushu Cancer Center, Fukuoka, Japan) from 2014 to 2018. Data from all of these patients were collected from medical charts and analyzed. The ages of the patients ranged from 42-87 years, and the mean and median ages were 68.3 years and 70 years, respectively. All patients basically sustained ability to perform certain activities of daily living, thus were categorized in Performance Status 0 or 1 by Eastern Cooperative Oncology Group scale. The co-morbidity scaled using Charlson's index (17) was $0 / 1$ in 70 patients (76\%) and $\geq 2$ in 22 patients $(24 \%)$. The patients were subcategorized into patients aged 79 years and younger (Group $A, n=79$ ), and 80 years and older (Group B, n=13). The mean/median ages of Group A and $\mathrm{B}$ were $65.9 / 68$ and $82.5 / 82$ years, respectively. The sex distribution was $57(62 \%)$ male and $35(38 \%)$ female. The disease was pancreatic tumor in 53 patients (58\%), bile duct tumor in $29(31 \%)$, and duodenal tumor in $10(11 \%)$. The Child method was applied for the reconstruction after PD. An internal or external stent was placed during the pancreatojejunostomy, and the abdominal cavity was routinely drained using closed suction tubes, in all patients. Six patients $(6 \%)$ underwent combined portal vein resection, $1(1 \%)$ underwent hepatic artery resection and reconstruction, and $1(1 \%)$ underwent partial hepatectomy. Postoperative pancreatic fistula and delayed gastric emptying were defined according to the International Study Group of Pancreatic Fistula $(18,19)$, and the grade of complication was defined by the Clavien-Dindo classification (20).

Immunological and nutritional assessment. Blood samples had been obtained within 1 week before surgery. Hematological parameters were determined immediately after blood sample collection, using an automatic blood analyzer. Parameters included white blood cell count, lymphocyte count, neutrophil count, monocyte count and platelet count $(12,21)$. The modified GPS was calculated from the serum albumin and serum C-reactive protein levels $(22,23)$. The PNI was calculated from the serum albumin level and the total lymphocyte count (24). Preoperative CONUT scores were calculated based on serum albumin concentrations, peripheral lymphocyte counts, and total cholesterol concentrations, as described elsewhere (16).

Body composition and physical functional assessment. Body composition and physical function assessments were performed by board certified physical therapists before surgery. The complete data was available and could be analyzed in 37 of the 92 patients, included 28 in Group A (79 years and younger) and 9 in Group B (80 years and older) patients. Body composition was determined using bioelectrical impedance analysis (BIA), with a TANITA MC780A model body analysis monitor (Tanita Inc., Tokyo, Japan). Skeletal muscle mass (SMM) was calculated with the following equation: $\mathrm{SMM}(\mathrm{kg})=0.566 \times$ fat free mass $(25)$. Skeletal muscle mass index (SMI) was calculated as skeletal muscle mass $(\mathrm{kg}) / \mathrm{height}$ squared (26). Cut-off thresholds for SMI were set at $7.0 \mathrm{~kg} / \mathrm{m}^{2}$ and $5.7 \mathrm{~kg} / \mathrm{m}^{2}$ for men and women, respectively (27). Handgrip strength (HGS) was measured to assess muscle strength, using a standard hand dynamometer. The cut-off thresholds for HGS were $18 \mathrm{~kg}$ for women and $26 \mathrm{~kg}$ for men (27). Walking speed was assessed via the $10 \mathrm{~m}$ walking test, and physical function was assessed using the 6-minute walking distance test (6MWD). The cut-off threshold for gait speed was $0.8 \mathrm{~m} / \mathrm{sec}(27)$.

Statistical analysis. All statistical analyses were performed using JMP 14 software (SAS; Cary, NC, USA). All variables were expressed as the mean \pm standard deviation. Continuous variables were compared using the Mann-Whitney $U$-test. Categorical data were compared using the $\chi^{2}$ test. Logistic regression analysis was performed to identify the independent predictors of complications. Survival outcomes were determined using the Kaplan-Meier method and compared by the log-rank test. A multivariate Cox proportional model was used to analyze independent prognostic factors in both overall survival and recurrence-free survival. A $p$-value of $<0.05$ was regarded as statistically significant.

\section{Results}

Preoperative immunonutritional condition. Preoperative immunonutritional status was compared between Group A (79 years and younger) and Group B (80 years and older). We used an established index that has been reported to be associated with short- and long-term prognosis after various surgeries $(16,22-24)$. As shown in Table I, preoperative serum albumin was significantly lower in Group B. PNI and CONUT scores, which include albumin in their calculations, were significantly lower in Group B. The NLR was significantly higher in Group B, but there was no statistically significant difference between the two groups in the plateletlymphocyte ratio and the lymphocyte-monocyte ratio. These data indicated a disturbed immunonutritional condition in the older patients.

Preoperative body composition and physical functional assessment. Body composition was assessed using BIA in 37 patients. The volumes and percentages of skeletal muscle were not different between the two groups, and thus SMI, 
Table I. Comparison of immunonutritional parameters.

\begin{tabular}{lcc}
\hline Factor & Group A $(\leq 79 \mathrm{y}, \mathrm{n}=79)$ & Group B ( $\geq 80 \mathrm{y}, \mathrm{n}=13)$ \\
\hline Body mass index & $22.3 \pm 0.3$ & $22.4 \pm 0.8$ \\
Serum albumin & $3.83 \pm 0.05$ & $3.61 \pm 0.12$ \\
Neutrophil-lymphocyte ratio & $2.32 \pm 0.13$ & $2.91 \pm 0.31$ \\
Platelet-lymphocyte ratio & $1.71 \pm 0.09$ & $1.69 \pm 0.23$ \\
Lymphocyte-monocyte ratio & $4.75 \pm 0.21$ & $4.40 \pm 0.52$ \\
Modified Glasgow prognostic score (0/1/2) & $59 / 15 / 5$ & $8 / 3 / 2$ \\
Prognostic nutrition index & $45.9 \pm 0.6$ & 0.046 \\
Controlling nutritional status (0-4/5-8) & $74 / 5(94 \% / 6 \%)$ & 0.469 \\
& & 0.272 \\
\end{tabular}

Table II. Comparison of body composition and functional assessment.

\begin{tabular}{lccc}
\hline Factor & Group A $(\leq 79 \mathrm{y}, \mathrm{n}=28)$ & Group B $(\geq 80 \mathrm{y}, \mathrm{n}=9)$ & $p$-Value \\
\hline Skeletal muscle volume $(\mathrm{kg})$ & $40.4 \pm 1.5$ & $36.8 \pm 2.6$ & 0.234 \\
Skeletal muscle percentage $(\%)$ & $71.1 \pm 0.01$ & $68.7 \pm 0.03$ & 0.236 \\
Skeletal muscle mass index (kg/m²) & $7.00 \pm 0.24$ & $6.72 \pm 0.43$ & 0.287 \\
Body fat percentage (\%) & $19.2 \pm 1.6$ & $23.7 \pm 2.8$ & 0.083 \\
Hand grip strength (kg) & $26.6 \pm 1.2$ & $61.5 \pm 2.2$ & 0.026 \\
Hand grip strength under cut-off & $5(15 \%)$ & $1.02 \pm 0.07$ & 0.007 \\
Gait speed (m/sec) & $1.30 \pm 0.04$ & $4(40 \%)$ & 0.0003 \\
Gait speed under cut-off & $2(6 \%)$ & $340 \pm 26$ & 0.013 \\
6-minute walk distance $(\mathrm{m})$ & $464 \pm 14$ & 0.0001 \\
\hline
\end{tabular}

Table III. Comparison of operative parameters and postoperative complications between elderly and non-elderly.

\begin{tabular}{|c|c|c|c|}
\hline Factors & Group A ( $\leq 79$ y, n=79) & Group B ( $\geq 80 \mathrm{y}, \mathrm{n}=13)$ & $p$-Value \\
\hline Operative time ( $\mathrm{min})$ & $347 \pm 10$ & $341 \pm 26$ & 0.425 \\
\hline Blood loss (g) & $391 \pm 40$ & $301 \pm 98$ & 0.211 \\
\hline Transfusion $(\%)$ & $3(4)$ & $0(0)$ & 0.517 \\
\hline \multicolumn{4}{|l|}{ Pancreatic texture } \\
\hline Soft/hard & $44 / 35$ & $6 / 7$ & 0.493 \\
\hline Pancreatic duct diameter (mm) & $3.6 \pm 0.2$ & $4.2 \pm 0.5$ & 0.231 \\
\hline \multicolumn{4}{|l|}{ Pancreaticojejunostomy stent } \\
\hline External/internal & $59 / 20$ & $7 / 6$ & 0.137 \\
\hline Incisional SSI (\%) & $8(10)$ & $2(15)$ & 0.589 \\
\hline Organ/space SSI (\%) & $45(57)$ & $7(54)$ & 0.834 \\
\hline Pancreatic fistula, Grade B/C (\%) & $24(30)$ & $5(38)$ & 0.567 \\
\hline Delayed gastric emptying (\%) & $13(16)$ & $3(28)$ & 0.992 \\
\hline Delirium (\%) & $2(3)$ & $5(38)$ & $<0.001$ \\
\hline Clavien-Dindo $\geq 3$ complication $(\%)$ & $20(25)$ & $5(38)$ & 0.983 \\
\hline Mortality (\%) & $1(1)$ & $0(0)$ & 0.683 \\
\hline
\end{tabular}

SSI: Surgical site infection.

the height-corrected skeletal muscle volume, was also similar between the groups (Table II). The body fat percentage tended to be higher in Group B (Table II, $p=0.083$ ). In contrast to body composition, a notable decrease of physical function was observed in the elderly group. HGS, gait speed, and 6MWD were significantly limited in the elderly patients (Table II). These results revealed that while the decrease of skeletal muscle volume was insignificant, the skeletal muscle function was dramatically impaired in the elderly compared to the non-elderly patients. 
Table IV. Risk factors associated with Clavien-Dindo Grade $\geq 3$ complications after pancreaticoduodenectomy.

\begin{tabular}{llcc}
\hline Factor & & hazard ratio (95\%CI) & $p$-Value \\
\hline Pancreatic texture & Soft/Hard & $18.4(2.18-155)$ & 0.008 \\
PNI & $\geq 46 /<46$ & $1.19(1.01-1.45)$ & 0.034 \\
Gender & Male/Female & $6.39(1.08-37.8)$ & 0.041 \\
CONUT & $\geq 5 / \leq 4$ & $17.1(0.83-355)$ & 0.066 \\
Preoperative cholangitis & $(+) /(-)$ & $4.97(0.89-27.9)$ & 0.068 \\
Curability & $0 / 1,2$ & $4.68(0.51-42.8)$ & 0.172 \\
Diabetes & $(+) /(-)$ & $2.16(0.50-9.39)$ & 0.304 \\
Charlson comorbidity index & $\geq 2 / \leq 1$ & $2.30(041-12.9)$ & 0.34 \\
Diagnosis & PDAC/others & $0.53(0.12-2.39)$ & 0.409 \\
Main pancreatic duct diameter (mm) & $\leq 3 / \geq 4$ & $1.91(0.36-10.0)$ & 0.444 \\
Age & $\geq 80 / \leq 79$ & $2.09(0.21-20.8)$ & 0.529 \\
Operative time (min) & $\geq 360 / \leq 359$ & $1.64(0.31-8.60)$ & 0.557 \\
BMI & $\geq 22 /<22$ & $1.49(0.39-5.67)$ & 0.559 \\
Smoking history & $(+) /(-)$ & $1.52(0.34-6.66)$ & 0.582 \\
NLR & $\geq 2.2 /<2.2$ & $1.17(0.29-4.71)$ & 0.826 \\
Blood loss (g) & $\geq 400 / \leq 399$ & $1.13(0.23-5.50)$ & 0.874 \\
\hline
\end{tabular}

BMI: Body mass index; CONUT: controlling nutritional score; CI: confidence interval; NLR: neutrophil-lymphocyte ratio; PDAC: pancreatic adenocarcinoma; PNI: prognostic nutritional index.

Comparison of short-term outcome of surgery. The intraoperative parameters were compared between Groups A and B. The mean operative times and mean intraoperative blood losses were comparable in Groups A and B, and their transfusion requirements did not differ (Table III). All patients underwent potentially curative surgery. Then, the short-term postoperative outcomes of the surgery were compared. The rates of morbidities such as surgical site infection, pancreatic fistula, and delayed gastric emptying were comparable in Groups A and B. The incidence of postoperative delirium was significantly higher in the elderly group $(p=0.0002)$. There was one postoperative death in Group A, for an overall mortality rate of $1.0 \%$.

Risk factors associated with morbidity after pancreaticoduodenectomy. Since the morbidity rates were comparable between the elderly and non-elderly patients who underwent PD, we investigated risk factors associated with postoperative complications of Clavien-Dindo Grade $\geq 3$ in all 92 patients. In multivariate analysis, soft pancreatic texture, high PNI (>46), and male sex were independent risk factors associated with postoperative complications (Table IV). High CONUT score and preoperative cholangitis tended be associated with postoperative complications $(p<0.07)$.

Long-term prognosis. The overall survival rates and recurrencefree survival rates were similar between Group A and Group B (data not shown). We also investigated the survival rates in patients with pancreatic cancer $(n=53)$, which revealed the same results. The multivariate analysis identified 3 factors that were prognostic for reduced overall survival (higher modified GPS index, higher CONUT score, and operative time $>360 \mathrm{~min}$ ) and one factor that was prognostic for reduced recurrence-free survival (postoperative complication, Clavien-Dindo Grade $\geq 3$ ).

\section{Discussion}

We showed that immunonutritional index and physical function were significantly impaired in the elderly patients who underwent PD, although the rates of postoperative complications were comparable between the elderly and nonelderly patients. These results indicated that the elderly are likely to have developed frailty and sarcopenia. Thus, a prehabilitation program including nutritional support and physiologic intervention is strongly warranted for the elderly before they undergo PD, to prevent postoperative complications.

The safety of PD for the elderly is still controversial among previous reports, which means it is unclear whether the indication of surgery can be determined by chronological age. Pancreatectomy is still the only possible curative treatment for pancreatic and periampullary cancers, and thus it is necessary to re-evaluate the indication of PD in the elderly, considering the recent progress of perioperative management. Our data showed that the age category of 80 years and older was not associated with mortality and morbidity after PD; instead, morbidity was associated with impaired immunonutritional index (low PNI). The PNI was significantly lower in the patients 80 years and older. These results indicated that elderly patients should not be excluded on the basis their calendar ages. However, they also indicated that the decision to perform PD can be made on the basis of the physical and immunonutritional reserve, without regard to age. 
Our data revealed that PNI and CONUT were significantly lower in the elderly who were to undergo PD, although these patients were predicted to be able to tolerate surgery. In addition to the nutritional index, NLR was also significantly higher in the elderly who were to undergo PD. A number of cohorts and meta-analyses supported the relationships between the immunonutritional index evaluated by laboratory data and long-term prognosis as well as postoperative complications in gastrointestinal and hepatopancreatobiliary (HPB) cancers $(28,29)$. The relevant biological mechanism relating the index and surgical outcomes has not been fully elucidated. However, it should be recognized that not only tumor-specific pathological factors, but also host-related nutritional status and systematic inflammatory response are responsible for the prognosis of patients with cancer. This study, to our knowledge, is the first to describe the relationship between aging and immunonutritional index in patients with HPB cancer.

It is well known that preoperative physical status, sarcopenia, comorbidities, and frailty are associated with surgical morbidity and mortality risk. Sarcopenia and frailty conceptually result from age-related decline in physiological reserve, and lead to disabilities, falls, fractures, and death (30). Our data showed that body composition and skeletal muscle amounts were comparable between the elderly and non-elderly subjects. These results may reflect that the patients were carefully selected to undergo PD, and the physical and nutritional prehabilitation protocols were introduced for the patients with sarcopenia and frailty to improve their physical condition in our series. Our data revealed that the physical condition, as evaluated by the grip strength and walking ability, was significantly impaired in the elderly, although body composition was comparable between the age groups. These results are worthy of attention because frailty caused by impaired physical activity has shown the most promise for identifying older adults at high risk of postoperative complications and nursing home discharge (31-33). We herein emphasize that physical activity, in addition to comorbidity, laboratory data and body composition, should be included in the preoperative geriatric assessment to decide on the operative indication for PD.

We acknowledge the limitations of this non-randomized study, but retrospective analysis is ethically and practically the only way to assess the eligibility of elderly patients for $\mathrm{PD}$, given the evidence that surgery is the only curative option for pancreatic and periampullary cancer (34). Several recent studies reported that prehabilitation can reduce overall and pulmonary morbidity following major abdominal surgery $(35,36)$. We recommend further studies employing the prehabilitation protocol for elderly patients who undergo PD, to assess the efficacy of workup and to establish the optimal protocols for specific operative procedures or specific patients.
In conclusion, the present study justified PD for the elderly with acceptable morbidity and prognosis. However, immunonutritional status and physical function were significantly impaired among that group. Thus, appropriate case selection and active nutritional support are required in the elderly.

\section{Conflicts of Interest}

K. Sugimachi and the co-authors have no conflicts of interest to declare regarding this study.

\section{Authors' Contributions}

Study conception and design: K Sugimachi; Acquisition of data: K Sugimachi, T Iguchi, Y Mano, M Ohta, M Ikebe; Analysis and interpretation of data: K Sugimachi, T Iguchi, Y Mano, T Nishijima; Drafting of manuscript: K Sugimachi, T Nishijima; Critical revision: M Morita, Y Toh.

\section{Acknowledgements}

The Authors thank Ms. Y. Urasawa and Ms. K. Toyokawa for technical assistance, Ms. E. Shindo for body composition and physical functional assessment. This study was supported in part by the Uehara Memorial Foundation, Tokyo, Japan.

\section{References}

1 Greenblatt DY, Kelly KJ, Rajamanickam V, Wan Y, Hanson T, Rettammel R, Winslow ER, Cho CS and Weber SM: Preoperative factors predict perioperative morbidity and mortality after pancreaticoduodenectomy. Ann Surg Oncol 18(8): 2126-2135, 2011. PMID: 21336514. DOI: 10.1245/s10434-011-1594-6

2 Gouma DJ, van Geenen RC, van Gulik TM, de Haan RJ, de Wit LT, Busch OR and Obertop H: Rates of complications and death after pancreaticoduodenectomy: Risk factors and the impact of hospital volume. Ann Surg 232(6): 786-795, 2000. PMID: 11088073. DOI: 10.1097/00000658-200012000-00007

3 Farnell MB, Nagorney DM and Sarr MG: The mayo clinic approach to the surgical treatment of adenocarcinoma of the pancreas. Surg Clin North Am 81(3): 611-623, 2001. PMID: 11459275. DOI: 10.1016/s0039-6109(05)70147-x

4 Yuan F, Essaji Y, Belley-Cote EP, Gafni A, Latchupatula L, Ruo $\mathrm{L}$ and Serrano PE: Postoperative complications in elderly patients following pancreaticoduodenectomy lead to increased postoperative mortality and costs. A retrospective cohort study. Int J Surg 60: 204-209, 2018. PMID: 30468904. DOI: 10.1016/j.ijsu.2018.11.016

5 Miyazaki Y, Kokudo T, Amikura K, Kageyama Y, Takahashi A, Ohkohchi N and Sakamoto H: Age does not affect complications and overall survival rate after pancreaticoduodenectomy: Singlecenter experience and systematic review of literature. Biosci Trends 10(4): 300-306, 2016. PMID: 27396698. DOI: 10.5582/ bst.2016.01093

6 Schnelldorfer T and Adams DB: The effect of malnutrition on morbidity after surgery for chronic pancreatitis. Am Surg 71(6): 466-472; discussion 472-463, 2005. PMID: 16044924. 
7 Peng P, Hyder O, Firoozmand A, Kneuertz P, Schulick RD, Huang D, Makary M, Hirose K, Edil B, Choti MA, Herman J, Cameron JL, Wolfgang CL and Pawlik TM: Impact of sarcopenia on outcomes following resection of pancreatic adenocarcinoma. J Gastrointest Surg 16(8): 1478-1486, 2012. PMID: 22692586. DOI: 10.1007/s11605-012-1923-5

8 Nishida Y, Kato Y, Kudo M, Aizawa H, Okubo S, Takahashi D, Nakayama Y, Kitaguchi K, Gotohda N, Takahashi S and Konishi M: Preoperative sarcopenia strongly influences the risk of postoperative pancreatic fistula formation after pancreaticoduodenectomy. J Gastrointest Surg 20(9): 1586-1594, 2016. PMID: 27126054. DOI: $10.1007 / \mathrm{s} 11605-016-3146-7$

9 Aida T, Furukawa K, Suzuki D, Shimizu H, Yoshidome H, Ohtsuka M, Kato A, Yoshitomi H and Miyazaki M: Preoperative immunonutrition decreases postoperative complications by modulating prostaglandin e 2 production and t-cell differentiation in patients undergoing pancreaticoduodenectomy. Surgery $155(1)$ : 124-133, 2014. PMID: 24589090. DOI: 10.1016/j.surg.2013. 05.040

10 Kitahata Y, Hirono S, Kawai M, Okada KI, Miyazawa M, Shimizu A, Kobayashi R, Ueno M, Hayami S, Shimokawa T, Kouda K, Tajima F and Yamaue H: Intensive perioperative rehabilitation improves surgical outcomes after pancreaticoduodenectomy. Langenbecks Arch Surg 403(6): 711-718, 2018. PMID: 30219924. DOI: 10.1007/s00423-018-1710-1

11 Souwer ETD, Bastiaannet E, de Bruijn S, Breugom AJ, van den Bos F, Portielje JEA and Dekker JWT: Comprehensive multidisciplinary care program for elderly colorectal cancer patients: "From prehabilitation to independence". Eur J Surg Oncol 44(12): 1894-1900, 2018. PMID: 30266205. DOI: 10.1016/j.ejso.2018.08.028

12 Mano Y, Shirabe K, Yamashita Y, Harimoto N, Tsujita E, Takeishi K, Aishima S, Ikegami T, Yoshizumi T, Yamanaka T and Maehara Y: Preoperative neutrophil-to-lymphocyte ratio is a predictor of survival after hepatectomy for hepatocellular carcinoma: A retrospective analysis. Ann Surg 258(2): 301-305, 2013. PMID: 23774313. DOI: 10.1097/SLA.0b013e318297ad6b

13 Kumamoto Y, Kaizu T, Tajima H, Nishizawa N, Ei S, Igarashi $\mathrm{K}$ and Watanabe $\mathrm{M}$ : Neutrophil-to-lymphocyte ratio as a predictor of postoperative morbidity in patients with distal cholangiocarcinoma. Mol Clin Oncol 9(4): 362-368, 2018. PMID: 30233792. DOI: $10.3892 / \mathrm{mco} .2018 .1698$

14 Nozoe T, Ninomiya M, Maeda T, Matsukuma A, Nakashima H and Ezaki T: Prognostic nutritional index: A tool to predict the biological aggressiveness of gastric carcinoma. Surg Today 40(5): 440-443, 2010. PMID: 20425547. DOI: 10.1007/s00595-009-4065-y

15 Shoji F, Morodomi Y, Akamine T, Takamori S, Katsura M, Takada K, Suzuki Y, Fujishita T, Okamoto T and Maehara Y: Predictive impact for postoperative recurrence using the preoperative prognostic nutritional index in pathological stage I non-small cell lung cancer. Lung Cancer 98: 15-21, 2016. PMID: 27393501. DOI: 10.1016/j.lungcan.2016.05.010

16 Harimoto N, Yoshizumi T, Inokuchi S, Itoh S, Adachi E, Ikeda Y, Uchiyama H, Utsunomiya T, Kajiyama K, Kimura K, Kishihara F, Sugimachi K, Tsujita E, Ninomiya M, Fukuzawa K, Maeda T, Shirabe K and Maehara Y: Prognostic significance of preoperative controlling nutritional status (conut) score in patients undergoing hepatic resection for hepatocellular carcinoma: A multiinstitutional study. Ann Surg Oncol 25(11): 3316-3323, 2018. PMID: 30051372. DOI: 10.1245/s 10434-018-6672-6
17 Charlson M, Peterson J, Szatrowski TP, MacKenzie R and Gold $\mathrm{J}$ : Long-term prognosis after peri-operative cardiac complications. J Clin Epidemiol 47(12): 1389-1400, 1994. PMID: 7730848. DOI: 10.1016/0895-4356(94)90083-3

18 Bassi C, Dervenis C, Butturini G, Fingerhut A, Yeo C, Izbicki J, Neoptolemos J, Sarr M, Traverso W, Buchler $M$ and International Study Group on Pancreatic Fistula D: Postoperative pancreatic fistula: An international study group (isgpf) definition. Surgery 138(1): 8-13, 2005. PMID: 16003309. DOI: 10.1016/j.surg.2005.05.001

19 Wente MN, Bassi C, Dervenis C, Fingerhut A, Gouma DJ, Izbicki JR, Neoptolemos JP, Padbury RT, Sarr MG, Traverso LW, Yeo CJ and Buchler MW: Delayed gastric emptying (dge) after pancreatic surgery: A suggested definition by the international study group of pancreatic surgery (isgps). Surgery 142(5): 761-768, 2007. PMID: 17981197. DOI: 10.1016/j.surg. 2007.05.005

20 Dindo D, Demartines $\mathrm{N}$ and Clavien PA: Classification of surgical complications: A new proposal with evaluation in a cohort of 6336 patients and results of a survey. Ann Surg 240(2): 205-213, 2004. PMID: 15273542. DOI: 10.1097/01.sla.000013 3083.54934.ae

21 Motomura T, Shirabe K, Mano Y, Muto J, Toshima T, Umemoto Y, Fukuhara T, Uchiyama H, Ikegami T, Yoshizumi T, Soejima Y and Maehara Y: Neutrophil-lymphocyte ratio reflects hepatocellular carcinoma recurrence after liver transplantation via inflammatory microenvironment. J Hepatol 58(1): 58-64, 2013. PMID: 22925 812. DOI: $10.1016 /$ j.jhep.2012.08.017

22 McMillan DC: The systemic inflammation-based glasgow prognostic score: A decade of experience in patients with cancer. Cancer Treat Rev 39(5): 534-540, 2013. PMID: 22995477. DOI: 10.1016/j.ctrv.2012.08.003

23 Toiyama Y, Miki C, Inoue Y, Tanaka K, Mohri Y and Kusunoki M: Evaluation of an inflammation-based prognostic score for the identification of patients requiring postoperative adjuvant chemotherapy for stage ii colorectal cancer. Exp Ther Med 2(1): 95-101, 2011. PMID: 22977476. DOI: 10.3892/etm.2010.175

24 Onodera T, Goseki N and Kosaki G: Prognostic nutritional index in gastrointestinal surgery of malnourished cancer patients. Nihon Geka Gakkai Zasshi 85(9): 1001-1005, 1984. PMID: 6438478.

25 Tsekoura M, Billis E, Tsepis E, Dimitriadis Z, Matzaroglou C, Tyllianakis M, Panagiotopoulos E and Gliatis $\mathrm{J}$ : The effects of group and home-based exercise programs in elderly with sarcopenia: A randomized controlled trial. J Clin Med 7(12), 2018. PMID: 30486262 . DOI: $10.3390 /$ jcm7120480

26 Bahat G, Tufan A, Tufan F, Kilic C, Akpinar TS, Kose M, Erten N, Karan MA and Cruz-Jentoft AJ: Cut-off points to identify sarcopenia according to european working group on sarcopenia in older people (ewgsop) definition. Clin Nutr 35(6): 1557-1563, 2016. PMID: 26922142. DOI: $10.1016 /$ j.clnu.2016.02.002

27 Chen LK, Liu LK, Woo J, Assantachai P, Auyeung TW, Bahyah KS, Chou MY, Chen LY, Hsu PS, Krairit O, Lee JS, Lee WJ, Lee Y, Liang CK, Limpawattana P, Lin CS, Peng LN, Satake S, Suzuki T, Won CW, Wu CH, Wu SN, Zhang T, Zeng $\mathrm{P}$, Akishita $\mathrm{M}$ and Arai $\mathrm{H}$ : Sarcopenia in asia: Consensus report of the asian working group for sarcopenia. J Am Med Dir Assoc 15(2): 95-101, 2014. PMID: 24461239. DOI: $10.1016 /$ j.jamda.2013.11.025 
28 Zhang Y and Zhang X: Controlling nutritional status score, a promising prognostic marker in patients with gastrointestinal cancers after surgery: A systematic review and meta-analysis. Int J Surg 55: 39-45, 2018. PMID: 29783000. DOI: 10.1016/j.ijsu. 2018.05.018

29 Takagi K, Domagala P, Polak WG, Buettner S and Ijzermans JNM: The controlling nutritional status score and postoperative complication risk in gastrointestinal and hepatopancreatobiliary surgical oncology: A systematic review and meta-analysis. Ann Nutr Metab 74(4): 303-312, 2019. PMID: 31013491. DOI: $10.1159 / 000500233$

30 Fried LP, Tangen CM, Walston J, Newman AB, Hirsch C, Gottdiener J, Seeman T, Tracy R, Kop WJ, Burke G, McBurnie MA and Cardiovascular Health Study Collaborative Research G: Frailty in older adults: Evidence for a phenotype. J Gerontol A Biol Sci Med Sci 56(3): M146-156, 2001. PMID: 11253156. DOI: $10.1093 /$ gerona/56.3.m146

31 Harimoto N, Yoshizumi T, Izumi T, Motomura T, Harada N, Itoh S, Ikegami T, Uchiyama H, Soejima Y, Nishie A, Kamishima T, Kusaba R, Shirabe K and Maehara Y: Clinical outcomes of living liver transplantation according to the presence of sarcopenia as defined by skeletal muscle mass, hand grip, and gait speed. Transplant Proc 49(9): 2144-2152, 2017. PMID: 29149975. DOI: 10.1016/j.transproceed.2017.09.017

32 Sato S, Nagai E, Taki Y, Watanabe M, Watanabe Y, Nakano K, Yamada H, Chiba T, Ishii Y, Ogiso H and Takagi M: Hand grip strength as a predictor of postoperative complications in esophageal cancer patients undergoing esophagectomy. Esophagus 15(1): 10-18, 2018. PMID: 29892804. DOI: 10.1007/ s10388-017-0587-3
33 Kapoor A, Matheos T, Walz M, McDonough C, Maheswaran A, Ruppell E, Mohamud D, Shaffer N, Zhou Y, Kaur S, Heard S, Crawford S, Cabral H, White DK, Santry H, Jette A, Fielding R, Silliman RA and Gurwitz J: Self-reported function more informative than frailty phenotype in predicting adverse postoperative course in older adults. J Am Geriatr Soc 65(11): 2522-2528, 2017. PMID: 28926087. DOI: 10.1111/jgs.15108

34 Shin SH, Park Y, Hwang DW, Song KB, Lee JH, Kwon J, Yoo C, Alshammary S, Kim SC: Prognostic Value of Adjuvant chemotherapy following pancreaticoduodenectomy in elderly patients with pancreatic cancer. Anticancer Res 39(2): 10051012, 2019. PMID: 30711988, DOI: 10.21873/anticanres.13206.

35 Hughes MJ, Hackney RJ, Lamb PJ, Wigmore SJ, Christopher Deans DA and Skipworth RJE: Prehabilitation before major abdominal surgery: A systematic review and meta-analysis. World J Surg, 2019. PMID: 30788536. DOI: 10.1007/s00268019-04950-y

36 Howard R, Yin YS, McCandless L, Wang S, Englesbe M and Machado-Aranda D: Taking control of your surgery: Impact of a prehabilitation program on major abdominal surgery. J Am Coll Surg 228(1): 72-80, 2019. PMID: 30359831. DOI: 10.1016/j.jamcollsurg.2018.09.018

Received September 20, 2019

Revised September 30, 2019

Accepted October 1, 2019 